Division of Cardiology, Department of Medicine, Weill Medical College of Cornell University, 525 East 68th Street, New York, New York 10021, USA. Phone: (212) 746-2201; Fax: (212) 746-2222; E-mail: ctbasson@med.cornell.edu.

1. Stauffer, B.L., Konhilas, J.P., Luczak, E.D., and Leinwand, L.A. 2006. Soy diet worsens heart disease in mice. J. Clin. Invest. 116:209-216. doi:10.1172/ JCI24676.

2. Maron, B.J., et al. 1995. Prevalence of hypertrophic cardiomyopathy in a general population of young adults. Echocardiographic analysis of 4111 subjects in the CARDIA Study. Coronary Artery Risk Development in (Young) Adults. Circulation. 92:785-789.

3. Davies, M.J., and McKenna, W.J. 1995. Hypertrophic cardiomyopathy-pathology and pathogenesis. Histopathology. 26:493-500.

4. Klues, H.G., Schiffers, A., and Maron, B.J. 1995. Phenotypic spectrum and patterns of left ventricular hypertrophy in hypertrophic cardiomyopathy: morphologic observations and significance as assessed by two-dimensional echocardiography in 600 patients. J. Am. Coll. Cardiol. 26:1699-1708.

5. Maron, B.J. 2002. Hypertrophic cardiomyopathy: a systematic review. J. Am. Med. Assoc. 287:1308-1320.
6. Maron, B.J., et al. 2003. American College of Cardiology/European Society of Cardiology clinical expert consensus document on hypertrophic cardiomyopathy. A report of the American College of Cardiology Foundation Task Force on Clinical Expert Consensus Documents and the European Society of Cardiology Committee for Practice Guidelines. J. Am. Coll. Cardiol. 42:1687-1713.

7. Spirito, P., Seidman, C.E., McKenna, W.J., and Maron, B.J. 1997. The management of hypertrophic cardiomyopathy. N. Engl. J. Med. 336:775-785.

8. Ahmad, F., Seidman, J.G., and Seidman, C.E. 2005 The genetic basis for cardiac remodeling. Annu. Rev. Genomics Hum. Genet. 6:185-216.

9. Horn-Ross, P.L., et al. 2001. Phytoestrogen consumption and breast cancer risk in a multiethnic population: the Bay Area Breast Cancer Study. Am. J. Epidemiol. 154:434-441.

10. Sirtori, C.R., Arnoldi, A., and Johnson, S.K. 2005. Phytoestrogens: end of a tale? Ann. Med. 37:423-438.

11. Cornwell, T., Cohick, W., and Raskin, I. 2004. Dietary phytoestrogens and health. Phytochemistry. 65:995-1016.

12. Squadrito, F., et al. 2002. The effect of the phytoestrogen genistein on plasma nitric oxide concentrations, endothelin-1 levels and endothelium dependent vasodilation in postmenopausal women. Atherosclerosis. 163:339-347.

13. Vega-Lopez, S., et al. 2005. Plasma antioxidant capacity in response to diets high in soy or animal protein with or without isoflavones. Am. J. Clin. Nutr. 81:43-49.

14. Dewell, A., Hollenbeck, C.B., and Bruce, B. 2002. The effects of soy-derived phytoestrogens on serum lipids and lipoproteins in moderately hypercholesterolemic postmenopausal women. J. Clin. Endocrinol. Metab. 87:118-121.

15. Vikstrom, K.L., Factor, S.M., and Leinwand, L.A. 1996. Mice expressing mutant myosin heavy chains are a model for familial hypertrophic cardiomyopathy. Mol. Med. 2:556-567.

16. Maass, A.H., Ikeda, K., Oberdorf-Maass, S., Maier, S.K., and Leinwand, L.A. 2004. Hypertrophy, fibrosis, and sudden cardiac death in response to pathological stimuli in mice with mutations in cardiac troponin T. Circulation. 110:2102-2109.

17. Dubey, R.K., Gillespie, D.G., Jackson, E.K., and Keller, P.J. 1998. 17Beta-estradiol, its metabolites, and progesterone inhibit cardiac fibroblast growth. Hypertension. 31:522-528.

18. Thigpen, J.E., et al. 1999. Phytoestrogen content of purified, open- and closed-formula laboratory animal diets. Lab. Anim. Sci. 49:530-536.

19. Iwasaki, K., et al. 1988. The influence of dietary protein source on longevity and age-related disease processes of Fischer rats. J. Gerontol. 43:B5-B12.

20. Shimokawa, I., et al. 1993. Diet and the suitability of the male Fischer 344 rat as a model for aging research. J. Gerontol. 48:B27-B32.

\title{
Gene expression profiling gets to the root of human hair follicle stem cells
}

\author{
George Cotsarelis
}

University of Pennsylvania School of Medicine, Philadelphia, Pennsylvania, USA.

\begin{abstract}
Hair follicle stem cells sustain growth and cycling of the hair follicle and are located in the permanent portion of the follicle known as the bulge. In this issue of the JCI, Ohyama et al. report the characterization of global gene expression patterns of human hair follicle stem cells after their isolation using sophisticated laser capture techniques to microdissect out bulge cells (see the related article beginning on page 249). They discovered a panel of cell surface markers useful for isolating living hair follicle stem cells, a finding with potential therapeutic implications since isolated stem cells in mice can generate new hair follicles when transplanted to other mice. The findings of Ohyama et al. validate the use of the mouse for studying hair follicle biology but also underscore critical differences between mouse and human stem cell markers. In particular, CD34, which delineates hair follicle stem cells in the mouse, is not expressed by human hair follicle stem cells, while CD200 is expressed by stem cells in both species. Ultimately, this information will assist efforts to develop cell-based and cell-targeted treatments for skin disease.
\end{abstract}

Advances in stem cell biology have resulted in major clinical benefits. Bone marrow transplantation for treatment

Nonstandard abbreviations used: K15, cytokeratin 15; LRC, label-retaining cell.

Conflict of interest: The author has declared that no conflict of interest exists.

Citation for this article: J. Clin. Invest. 116:19-22 (2006). doi:10.1172/JCI27490. of cancers and corneal transplantation for treatment of blindness resulting from chemical burns were both possible because stem cells were identified and isolated from the affected tissues $(1,2)$. Many disorders of the skin, such as cancer, chronic wounds, skin atrophy and fragility, hirsutism, and alopecia, can be viewed as disorders of adult stem cells. Because stem cells in the epidermis and hair follicle serve as the ultimate source of cells for both of these tissues, understanding the control of their proliferation and differentiation is key to understanding disorders related to disruption in these processes. Furthermore, the isolation, cultivation, and propagation of epithelial stem cells are important for tissue-engineering approaches to treating skin disorders (3).

The skin possesses an outer covering produced by the epidermis that protects us from dehydration and from external environmental insults. The outer layer of the hair follicle is contiguous with the epidermis, forming invaginations that, in the case of scalp follicles, penetrate deeply into the fat underlying the skin. Approximately 5 million follicles that are spread over the body generate hair in a cyclical fashion. The duration of anagen (the period of hair growth), which varies from less than 60 to more than several thousand days on different body sites, determines the length of the hair shaft. After anagen, the follicle enters a stage of involution (catagen) and then a stage of 


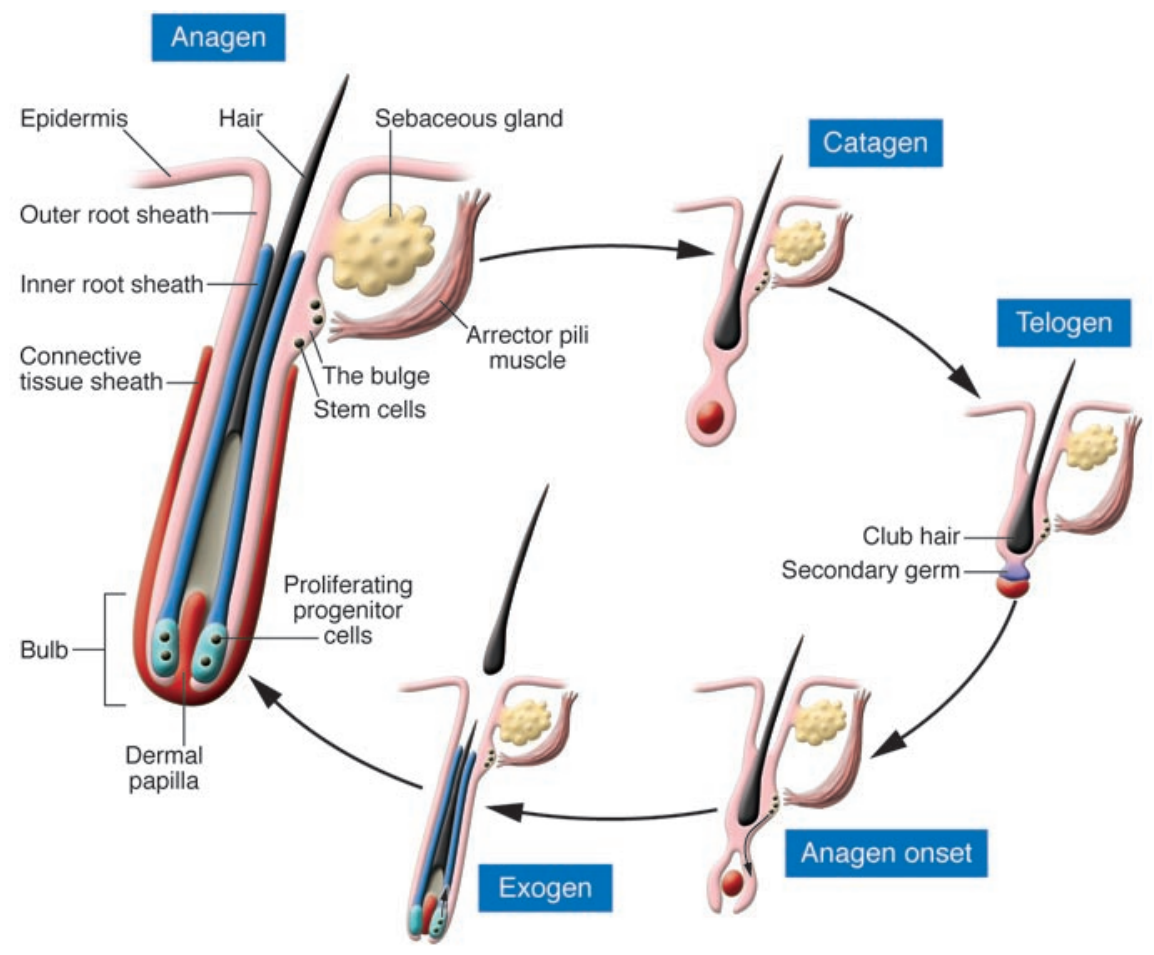

Figure 1

Hair follicle cycle. Cyclical changes in hair follicle growth are divided into different stages, referred to as anagen, catagen, telogen, and exogen. Hair follicle stem cells localize to the bulge at the bottom of the permanent follicle at the site of arrector pili muscle attachment. During anagen, rapidly proliferating progenitor cells in the bulb generate the hair and its surrounding inner root sheath. The onset of catagen is marked by cessation of proliferation and apoptosis of the epithelial cells below the bulge. The mesenchymally derived dermal papilla survives catagen and moves upward to abut the lowermost portion of the bulge, which then forms the secondary germ at its base, during telogen. III-defined mesenchymal-epithelial interactions likely involving BMP and WNT signaling are thought to signal anagen onset and the generation of the new lower follicle and hair shaft. As the new hair grows in, the old hair is shed during exogen. The duration of each stage varies depending on the type, site, and genetic programming of the follicle.

rest (telogen), when the hair that was produced is now dead yet remains anchored in the follicle until it is shed during exogen (Figure 1).

Eventually, stem cells at the base of the resting follicle, in an area known as the bulge, proliferate and regenerate a new lower follicle including the highly proliferative bulb cells that produce a new hair (Figure 1). After giving rise to the new hair-producing cells, the stem cells return to a quiescent state that is a hallmark of these cells. Despite being surrounded by highly proliferating epidermal and hair follicle cells, the cells in the bulge rarely undergo mitosis. The extended state of dormancy is in line with other stem-like characteristics of these cells, including a prolonged lifespan - probably as long as that of the organism. This characteristic is thought to predispose these cells to by cavernous blood-filled sinuses and are innervated by large nerves that track to the brain cortex and are organized into large "barrel fields," each corresponding to one vibrissa follicle. Because the primary purpose of vibrissa follicles is tactile sensation (known as whisking), they do not cycle in the same manner as other follicles. Rather than entering a true resting phase, these follicles only briefly stop proliferating, without regressing, before generating a new hair shaft. The old shaft is retained in the follicle until the new shaft comes in beside it so that the follicle is never devoid of a long hair, and thus the animal maintains its sensory perception. This stands in stark contrast to human follicles, which undergo dramatic shortening, losing up to $80 \%$ of their lower volume during catagen, the stage of regression (Figure 1), and also possess only 1 hair per follicle, while growing for years rather than weeks. Thus, the cellular and molecular characteristics of stem cells in the human follicle could be quite different from those in the rodent.

\section{Identification of stem cells}

Putative stem cells in mouse pelage (back skin) and vibrissa follicles were previously identified as quiescent cells in vivo by label-retaining cell (LRC) studies (5). Bulge cells in the adult mouse were then shown, through genetic labeling studies, to generate all of the cell lineages within the follicle in vivo and after isolation, and thus to function as stem cells (4). By transplantation of human scalp tissue to immunodeficient mice, LRCs in human follicles were localized to the basal layer of the outer root sheath in the area of the bulge (9). Because the human bulge is not morphologically apparent, markers were needed for the study of this area. Cytokeratin 15 (K15), an intracellular intermediate filament protein, was identified as one marker preferentially expressed by the human bulge (9). Ideally, however, cell surface proteins against which antibodies could be raised would be valuable for isolating the human bulge cells, testing their stem cell characteristics, and defining global gene expression patterns important for understanding control mechanisms for stem cell growth and proliferation.

In this issue of the JCI, Ohyama et al. (10) report having defined the bulge area of the human hair follicle using a combination of proliferative characteristics, 


\section{Table 1}

Selected genes upregulated in bulge cells compared with nonbulge epithelial cells

\begin{tabular}{lcc}
\hline & Human & Mouse \\
CD34 & - & + \\
CD200 & + & + \\
K15 & + & + \\
ID2 & + & + \\
DKK3 & + & + \\
WIF1 & + & + \\
FZD1 or FZD2 & + & + \\
PHLDA1 & + & - \\
FOLLISTATIN & + & - \\
DIO2 & + & - \\
CD71 & - & - \\
\hline
\end{tabular}

Human and mouse bulge cells preferentially express many of the same genes, but critical differences were also discovered, as reported in this issue of the $\mathrm{JCl}$ by Ohyama et al. (10). In general, inhibitors of the WNT pathway, which is important for hair follicle cycling and differentiation, are increased. ID2, a gene previously associated with relatively undifferentiated cells, is upregulated in both species. Surprisingly, CD34, which is a specific bulge cell marker in the mouse, is lacking in human follicle stem cells. FOLLISTATIN and $\mathrm{DIO} 2$ are both in human but not mouse hair follicle bulge cells, and this may indicate their possible roles as mediators of hormonal pathways.

histological landmarks, and biochemical features. The location of quiescent LRCs in human scalp tissue grafted to immunodeficient mice was compared with the location of attachment of the arrector pili muscle (responsible for "goose bumps" upon contraction), which was identified using antibodies against smooth muscle. The LRCs were found at and above the site of muscle insertion up to the level of the sebaceous gland (Figure 1). The area containing the LRCs was also positive for $\mathrm{K} 15$, in contrast to the remainder of the outer root sheath, which is generally negative for this marker. K15 expression correlates with an immature phenotype within the skin, since it occurs in neonatal but not adult interfollicular epidermal cells (11), and thus reflects the distinct biochemical makeup of the bulge cells compared with the rest of the epidermis and hair follicle.

\section{Searching for stem cell genes}

Defining gene expression patterns that control the quiescent, noncycling nature of hair follicle stem cells is a major goal in epithelial stem cell biology, since it could lead to a better understanding of the abnormal proliferation that these cells undergo in the development of skin cancer (4). In addition, elucidation of genes involved in hair follicle stem cell proliferation and self-renewal may provide insights into alopecia and abnormal wound healing, since bulge cells are important for hair growth and normal wound healing (12). In order to identify genes related to the stem cell phenotype, Ohyama et al. (10) compared microarray gene expression profiles of bulge cells with those of other closely related cell populations within the hair follicle. Unlike in many other microarray studies in which heterogeneous mixtures of cell types were used to generate mRNA, the use of laser capture microdissection, in which individual cells or groups of cells are dissected out under a microscope from tissue sections on a glass slide and captured using a laser, permitted isolation of a specific population of mRNA highly enriched for messages found in stem cells. Ohyama et al. discovered the presence of many of the same differentially expressed genes in the human bulge cells that were previously described in mouse bulge cells $(4,8)$; however, several key differences between the mouse and the human data were noted (Table 1). In particular, CD34, which is an excellent bulge cell marker in the mouse (13), is not present on human bulge cells. Rather, CD34 is expressed in slightly more differentiated outer root sheath cells below the bulge in the human follicle; therefore, antibodies against CD34 cannot be used to isolate human hair follicle stem cells. To circumvent this problem, Ohyama et al. (10) identified a panel of bulge stem cell surface markers, including some previously identified in the mouse (e.g., CD200; ref. 4). Using a combination of antibodies against these markers as well as negative markers for nonbulge cells, human hair follicle stem cells were successfully isolated and then propagated in culture, a feat that is a prerequisite to using these cells for tissue-engineering purposes and that up to now has been technically impossible (3).

\section{Outside influences}

The stem cell microenvironment or niche in the bulge appears to possess potent signals for maintaining stem cells in an undifferentiated state. In addition to epi- thelial stem cells, the bulge also contains other stem cell types, including melanocyte stem cells (14). That the stem cell niche is a unique microenvironment is supported by several lines of evidence. Interestingly, after an injury to the bulge cells causes apoptosis, the bulge can be repopulated by cells that are slightly more differentiated and reside at the base of the follicle, called the secondary germ (15). Presumably this is a function of the microenvironment in an effort to maintain the integrity of the bulge area by actually inducing cells that are direct descendents of stem cells to revert to a stem cell phenotype. Furthermore, the power of the niche evidently may instruct bone marrow-derived dendritic APCs to remain relatively undifferentiated (16), and even influence mast cells to remain in a precursor state (17).

The findings reported by Ohyama et al. (10) suggest that CD200 is an important regulator of the immune-privileged state of the hair follicle. This is in line with a previous study demonstrating that CD200 inhibits autoimmune inflammation in the hair follicle (18). This suggests that certain types of alopecia, such as lichen planopilaris, discoid lupus erythematosus, alopecia areata, and even androgenetic alopecia, with which inflammation appears to be associated, eventually could be treated with strategies aimed at augmenting CD200 expression.

The use of mouse models for studying human biology has been validated by these studies, with the caveat that findings from mouse and other animal models require confirmation in human systems. Because Ohyama et al.'s findings (10) are derived directly from human tissue, the significance of this line of research is greatly enhanced. By defining unique sets of cell surface markers for adult stem cells and other cell populations within the human follicle, investigators can now readily extract and study very specific follicular cell types for their ability to regenerate hair and skin, with the hope of developing novel methods for treating skin and hair disease.

Address correspondence to: George Cotsarelis, University of Pennsylvania School of Medicine, M8 Stellar-Chance Laboratories, 422 Curie Boulevard, Philadelphia, Pennsylvania 19104, USA. Phone: (610) 902-2400; Fax: (215) 573-9102; E-mail: cotsarel@mail.med.upenn.edu. 
1. Bernstein, I.D., Andrews, R.G., and Rowley, S. 1994 Isolation of human hematopoietic stem cells. Blood Cells. 20:15-24.

2. Tsai, R.J., Li, L.M., and Chen, J.K. 2000. Reconstruction of damaged corneas by transplantation of autologous limbal epithelial cells. N. Engl. J. Med. 343:86-93.

3. Stenn, K.S., and Cotsarelis, G. 2005. Bioengineering the hair follicle: fringe benefits of stem cell technology. Curr. Opin. Biotechnol. 16:493-497.

4. Morris, R.J. 2000. Keratinocyte stem cells: targets for cutaneous carcinogens. J. Clin. Invest. 106:3-8.

5. Cotsarelis, G., Sun, T.T., and Lavker, R.M. 1990 Label-retaining cells reside in the bulge area of pilosebaceous unit: implications for follicular stem cells, hair cycle, and skin carcinogenesis. Cell. 61:1329-1337.

6. Morris, R.J., et al. 2004. Capturing and profiling adult hair follicle stem cells. Nat. Biotechnol. 22:411-417.

7. Oshima, H., Rochat, A., Kedzia, C., Kobayashi, K., and Barrandon, Y. 2001. Morphogenesis and renewal of hair follicles from adult multipotent stem cells. Cell. 104:233-245.

8. Tumbar, T., et al. 2004. Defining the epithelial stem cell niche in skin. Science. 303:359-363.

9. Lyle, S., et al. 1998. The C8/144B monoclonal antibody recognizes cytokeratin 15 and defines the location of human hair follicle stem cells. J. Cell Sci. 111:3179-3188.

10. Ohyama, M., et al. 2006. Characterization and isolation of stem cell-enriched human hair follicle bulge cells. J. Clin. Invest. 116:249-260. doi:10.1172/ JCI26043.

11. Liu, Y., Lyle, S., Yang, Z., and Cotsarelis, G. 2003. Keratin 15 promoter targets putative epithelial stem cells in the hair follicle bulge. J. Invest. Dermatol. 121:963-968.

12. Ito, M., et al. 2005. Stem cells in the hair follicle bulge contribute to wound healing but not to homeostasis of the epidermis. Nat. Med. 11:1351-1354.

13. Trempus, C., et al. 2003. Enrichment for living murine keratinocytes from the hair follicle bulge with the cell surface marker CD34. J. Invest. Dermatol. 120:501-511.

14. Nishimura, E.K., et al. 2002. Dominant role of the niche in melanocyte stem-cell fate determination. Nature. 416:854-860.

15. Ito, M., Kizawa, K., Toyoda, M., and Morohashi, M. 2002. Label-retaining cells in the bulge region are directed to cell death after plucking, followed by healing from the surviving hair germ. J. Invest. Dermatol. 119:1310-1316.

16. Gilliam, A.C., et al. 1998. The human hair follicle: a reservoir of CD40+ B7-deficient Langerhans cells that repopulate epidermis after UVB exposure. J. Invest. Dermatol. 110:422-427.

17. Kumamoto, T., et al. 2003. Hair follicles serve as local reservoirs of skin mast cell precursors. Blood. 102:1654-1660.

18. Rosenblum, M.D., et al. 2004. Expression of CD200 on epithelial cells of the murine hair follicle: a role in tissue-specific immune tolerance? J. Invest. Dermatol. 123:880-887.

\section{Delving deeper into MALT lymphoma biology}

\section{Francesco Bertoni and Emanuele Zucca}

Laboratory of Experimental Oncology and Lymphoma Unit, Oncology Institute of Southern Switzerland, Bellinzona, Switzerland.

\begin{abstract}
Mucosa-associated lymphoid tissue (MALT) lymphomas can arise in a variety of extranodal sites. Interestingly, at least 3 different, apparently site-specific, chromosomal translocations, all affecting the NF- $\kappa B$ pathway, have been implicated in the development and progression of MALT lymphoma. The most common is the translocation $t(11 ; 18)(q 21 ; q 21)$, which results in a fusion of the cIAP2 region on chromosome $11 \mathrm{q} 21$ with the MALT1 gene on chromosome 18q21 and is present in more than onethird of cases. The frequency of this translocation is site-related: common in the gastrointestinal tract and lung, rare in conjunctiva and orbit, and almost absent in salivary glands, thyroid, liver, and skin. In this issue of the JCI, Hu et al. add to our understanding of the molecular consequences of this translocation, showing that its fusion product, cIAP2MALT1, may concomitantly contribute to lymphomagenesis both as a tumor suppressor gene and as an oncogene (see the related article beginning on page 174).
\end{abstract}

\section{MALT Iymphoma: \\ the clinical background}

Extranodal marginal zone B cell lymphoma (EMZL) of mucosa-associated lymphoid tissue (MALT), also known as MALT lymphoma, is a neoplastic disease that constitutes approximately $8 \%$ of all nonHodgkin lymphomas (1-4). It can arise in a variety of extranodal sites and occurs

Nonstandard abbreviations used: BIR, baculoviral IAP repeat; CARD, caspase recruitment domain; cIAP2, cellular inhibitor of apoptosis protein 2 ; DD, death domain; EMZL, extranodal marginal zone B cell lymphoma; IAP, inhibitor of apoptosis protein; IкB, inhibitory $\kappa \mathrm{B}$; IKK, IкB kinase; MALT, mucosa-associated lymphoid tissue; NES, nuclear export signal.

Conflict of interest: The authors have declared that no conflict of interest exists.

Citation for this article: J. Clin. Invest. 116:22-26 (2006). doi:10.1172/JCI27476. most often in organs such as the stomach, the salivary glands, or the thyroid, where lymphocytes are normally absent. MALT is the result of chronic phlogistic events in response to either infectious conditions such as Helicobacter pylori-associated chronic gastritis, or autoimmune disorders like Hashimoto thyroiditis and myoepithelial sialadenitis. In these conditions abnormal B cell clones can progressively replace the normal B cell population of the inflammatory tissue, giving rise to the EMZL. Over 10 years ago, $H$. pylori was identified as an etiologic factor in gastric MALT lymphomas after the demonstration of tumor regression in the majority of early-stage cases treated with anti-Helicobacter antibiotic therapy, and this tumor therefore became a popular model of the close pathogenetic link between chronic inflammation and lymphoma development. Other bacterial infections were later possibly implicated in the pathogenesis of MALT lymphomas arising in the skin (Borrelia burgdorferi), in the ocular adnexa (Chlamydia psittaci), and in the small intestine (Campylobacter jejuni) $(1,4)$.

The prognosis for patients with MALT lymphomas is good; these tumors usually have an indolent course with overall 5-year survival rates greater than $80 \%$, but rare cases with histologic transformation in cases of aggressive diffuse large-cell lymphoma have been described. It is nowadays generally accepted that eradication of $H$. pylori with antibiotics should be used as the sole initial treatment of localized gastric MALT lymphoma, while the use of anti-infectious treatment in nongastric locations is still under investigation. Other effective treatment approaches include radiotherapy, chemotherapy, and antiCD20 mAbs $(2,3)$.

\section{Many chromosomal translocations affecting the same pathway}

Four main recurrent chromosomal translocations have been associated with the pathogenesis of EMZLs: $t(11 ; 18)(\mathrm{q} 21 ; \mathrm{q} 21)$, $\mathrm{t}(1 ; 14)(\mathrm{p} 22 ; \mathrm{q} 32), \mathrm{t}(14 ; 18)(\mathrm{q} 32 ; \mathrm{q} 21)$, and $\mathrm{t}(3 ; 14)(\mathrm{p} 14.1 ; \mathrm{q} 32)(5-8)$ (Table 1). The latter is the most recently described and establishes the juxtaposition of the transcription factor FOXP1 next to the enhancer region 